\title{
Journal of Endocrinology and Metabolism Research
}

Karunakaran V, et al., 2020- J Endo Metabol Res

\section{Monogenic Diabetes due to ABCC8/KCNJ11 Mutation: Case Study and Review of Literature}

\author{
Karunakaran $\mathrm{V}^{\mathbf{1}}$, Wei $\mathrm{C}^{\mathbf{2}}$, and Bano $\mathrm{G}^{\mathbf{1}}$
}

\begin{abstract}
Monogenic diabetes arises due to mutation in a single-gene and is recognized by their striking familial inheritance pattern. This form of diabetes is inherited in an autosomal dominant or recessive fashion, unlike polygenic Type 1 (autoimmune) or type 2 diabetes caused by the combined action of more than one gene [1-11]. Monogenic diabetes is classified into three main groups: Neonatal diabetes mostly presents in the first six months of birth, maturity onset diabetes of the young (MODY) and maternally inherited mitochondrial diabetes.

These mutations run in the family and have a predictable course. Most of the monogenic diabetes is treated with oral medications like sulfonylurea rather than insulin. ABCC8/KCNJ11 gene mutations also cause monogenic diabetes. This gene mutation has been found in $\sim 50 \%$ of congenital hyperinsulinemia (CHI) patients. In such cases diabetes commonly presents in the neonatal period (transient or permanent) or at adolescence / early adulthood [1].

We present a 58-year-old diabetic lady, who was detected with ABCC 8 mutation during the cascade testing [8]. She was diagnosed with diabetes at the age of 12 [8]. Her son had history of neonatal hypoglycaemia and developed diabetes at the age of 15 . He was the index case who was found to have ABCC8 mutation. The family has several other members diagnosed with diabetes. The aim of the article is to increase awareness and understanding of monogenic diabetes among the medical practitioners in adult population with diabetes so that the genetic testing can be offered in a cost effective manner.
\end{abstract}

Keywords: Monogenic Diabetes; Neonatal Diabetes; Maturity Onset Diabetes of the Young; Maternally Inherited Mitochondrial Diabetes; Congenital Hyperinsulinemia

\section{Introduction}

The physiological regulation of insulin release from the pancreatic beta-cells is now fairly well understood. Understanding the molecular basics of Insulin secretion from the pancreatic beta-cell in response to the rise in plasma glucose has paved the way for identifying a variety of mutations causing monogenic diabetes. In $\beta$ - cells, the main stimulus for insulin release is elevated blood glucose levels following a meal. Under physiological circumstances, the postprandial plasma glucose enters the 
pancreatic beta-cell through GLUT2 ( $\left.\mathrm{SLC}_{2} \mathrm{~A}_{2}\right)$, transporter, which is located on the beta-cell surface. Glucose is then phosphorylated by Glucokinase enzyme to glucose-6-phosphate, and this enters the Krebs cycle, causing increased ATP production. The change in ATP: ADP ratio causes the closure of Potassium sensitive Adenosine-Tri- Phosphate (KATP) channels, resulting in membrane depolarization and opening of the Voltagegated Calcium channels with a resultant influx of extracellular Calcium ion into the pancreatic beta-cell. This influx leads to degranulation of insulin-containing vesicles and Insulin release. Figure 1 explains the physiology of Insulin release from a pancreatic beta-cell and the common mutations involved in causing monogenic diabetes [2].

Currently, there are at least 41 identified genes involved in monogenic diabetes (Table 1) [1]. The commonest 4 causes of MODY are MODY2 (GCK) and $\mathrm{MODY}_{3}$ (HNFIA), MODY1 (HNF4A) and MODY5 (HNF1B). MODY2 and MODY 3 account for 30\%-60\% of all MODY, whereas, MODY 1 and MODY 5 together account for $10 \%$ of all MODY. Pathogenic variation in 10 other genes can result in $20 \%$ of all MODY [1]. A substantial number of MODY may be caused by variation in un-identified genes or could not be identified by the old genetic testing methods [3].

ABCC8 (ATP-binding cassette transporter, sub-family C, member 8) and KCNJu (Potassium inwardly rectifying channel, subfamily J, member 11) gene mutations constitute $40 \%$ of neonatal diabetes. They result in either gain of function or loss of function mutation of the KATP channel, but evidence indicates that the majority are due to gain of function mutation. With a gain of function mutation, the KATP channel remains open, and there is no insulin release resulting in diabetes Figure 2. With the loss of function mutation, the KATP channel is not manufactured, or it is formed, but trapped in the endoplasmic reticulum Figure 3, or it traffics to the cell surface but is unresponsive to mgADP thus resulting in its closure and dysregulated insulin release Figure 4. This causes congenital hyperinsulinemia and the resultant hypoglycemia during the neonatal period $[4,5]$. In pancreatic b cells glucose release requires $\mathrm{Ca}^{+}$influx Low blood glucose: Decrease ATP-Increase $\mathrm{K}^{+}$ efflux-increase Increase depolarizationdecrease $\mathrm{Ca}^{2+}$ influx and decrease insulin secretion

Elevated blood glucose: Increase ATPdecrease $\mathrm{K}^{+}$efflux, decrease Depolarization, increase $\mathrm{Ca}^{2+}$ influx and increase insulin secretion.

Prevents trafficking KATP channel Membrane stays in depolarized state regardless of metabolic state Results in dysregulated insulin secretion. The nextgeneration sequencing technology analyses the pathogenic variants, increasing the chances of detection of several novel pathological Variants of Unknown Significance (VUS). This technology also raises ethical and clinical dilemmas that deserve a careful and conscientious interpretation, often with a multidisciplinary (MDT) approach with multi-center collaboration $[1,6]$. 
Table 1: Maturity-Onset Diabetes of the Young (MODY): Associate Genes and Clinical Features

\begin{tabular}{|c|c|c|c|}
\hline Gene & $\begin{array}{l}\text { \%age of } \\
\text { diabetes }\end{array}$ & Clinical Features & $\begin{array}{l}\text { Microvascular } \\
\text { complications }\end{array}$ \\
\hline $\mathrm{HNF}_{4} \mathrm{~A}(\mathrm{MODY} \mathbf{1})$ & $5-10 \%$ & $\begin{array}{l}\text { Birth weight is usually more than 8oogm } \\
\text { Neonates may develop transient } \\
\text { hyperinsulinemichypoglycemia Defect in } \\
\text { insulin secretion tends to be progressive }\end{array}$ & Common \\
\hline GCK (MODY2) & $30-50 \%$ & $\begin{array}{c}\text { Mild fasting hyperglycemia at birth } \\
\text { Mostly asymptomatic } \\
\text { Often incidental diagnosis }\end{array}$ & Rare \\
\hline $\mathrm{HNF}_{1} \mathrm{~A}\left(\mathrm{MODY}_{3}\right)$ & $30-65 \%$ & $\begin{array}{l}\text { Some cases present with transient } \\
\text { neonatal hyperinsulinemichypoglycemia } \\
\text { Insulin secretory defect is progressive } \\
\text { Renal glycosuria is present } \\
\text { Oral glucose tolerance test (OGTT) is } \\
\text { required to establish early diagnosis }\end{array}$ & Common \\
\hline PDX1 $1\left(\mathrm{MODY}_{4}\right)$ & $1 \%$ & Overweight or obesity in some cases & Not known \\
\hline HNF1B (MODY 5$)$ & $5 \%$ & $\begin{array}{c}\text { Intrauterine growth retardation (IUGR) } \\
\text { Renal and urogenital tract } \\
\text { anomalies Hypoplastic Pancreas }\end{array}$ & Common \\
\hline $\begin{array}{l}\text { NEUROD1 } \\
\text { (MODY6) }\end{array}$ & $1 \%$ & Some case may be overweight or obese & Not known \\
\hline KLF11 (MODY7) & $\begin{array}{l}\text { Less than } \\
\quad 1 \%\end{array}$ & & Not known \\
\hline \multirow[b]{2}{*}{ CEL (MODY8) } & \multirow{2}{*}{$\begin{array}{l}\text { Less than } \\
\quad 1 \%\end{array}$} & Atrophy of Pancreas & \multirow[b]{2}{*}{ Rare } \\
\hline & & $\begin{array}{c}\text { Pancreatic exocrine insufficiency } \\
\text { Lipomatosis and Fibrosis }\end{array}$ & \\
\hline $\mathrm{PAX}_{4}$ (MODY9) & $\begin{array}{l}\text { Less than } \\
\qquad 1 \%\end{array}$ & & Not known \\
\hline INS1 (MODY1o) & $\begin{array}{l}\text { Less than } \\
\qquad 1 \%\end{array}$ & & Not known \\
\hline BLK (MODY11) & $\begin{array}{l}\text { Less than } \\
\quad 1 \%\end{array}$ & Some case may be overweight or obese & Not known \\
\hline ABCC8 (MODY12) & $\begin{array}{l}\text { Less than } \\
\quad 1 \%\end{array}$ & Same as $\mathrm{HNF}_{1} \mathrm{~A}$ and $\mathrm{HNF}_{4} \mathrm{~A}$ & Not known \\
\hline $\begin{array}{c}\text { KCNJ112 } \\
\left(\text { MODY13) }^{2}\right.\end{array}$ & $\begin{array}{l}\text { Less than } \\
\quad 1 \%\end{array}$ & Same as $\mathrm{HNF}_{1} \mathrm{~A}$ and $\mathrm{HNF}_{4} \mathrm{~A}$ & Not known \\
\hline APPLi ( MODY14) & $\begin{array}{l}\text { Less than } \\
\qquad 1 \%\end{array}$ & Some case may be overweight or obese & Not known \\
\hline
\end{tabular}

Figure 1: Pancreatic b-cell glucose detection and Insulin release in a normal cell. 


\section{Pancreatic $\beta$-cell glucose detection and insulin release}

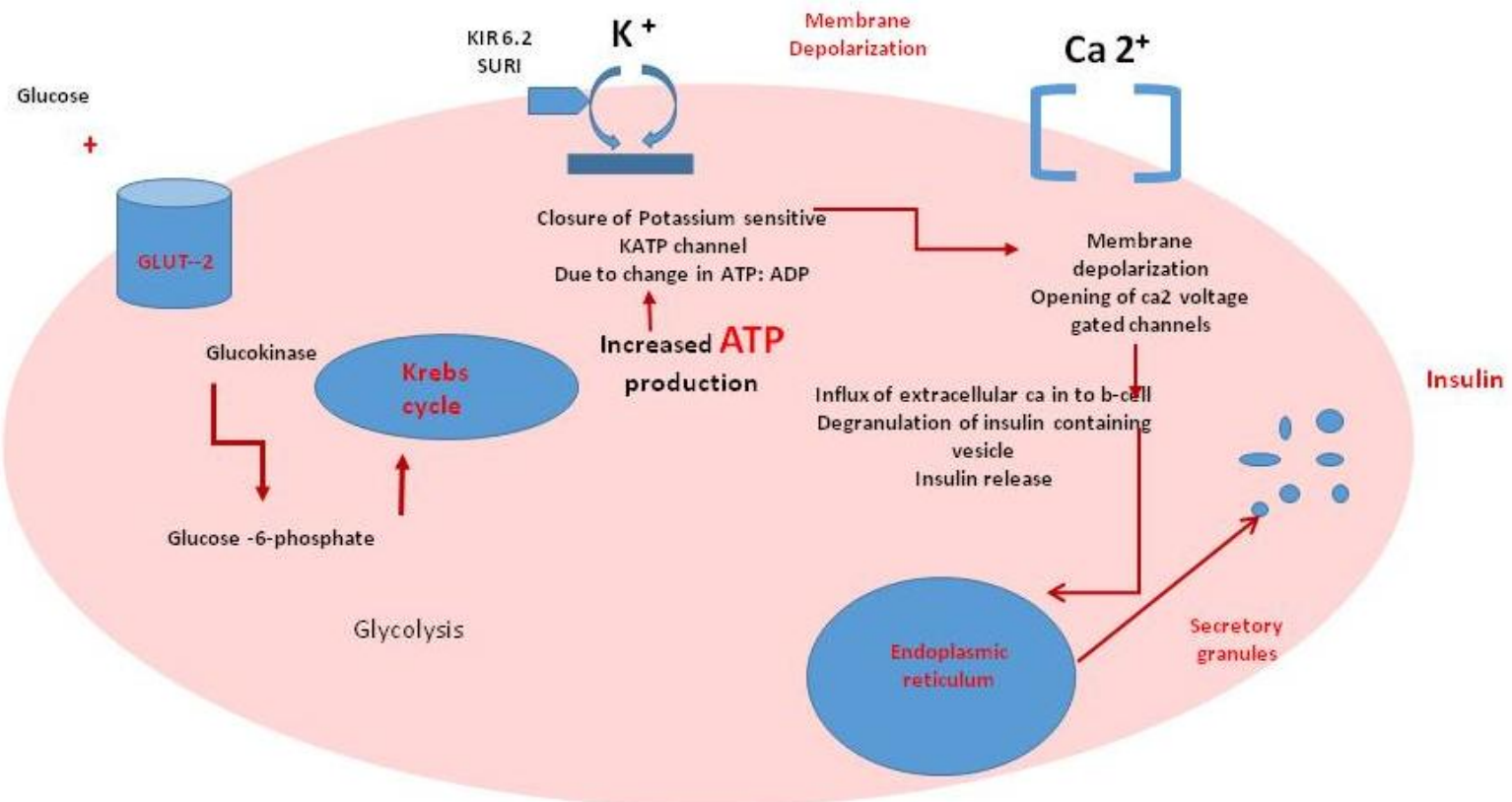

Figure 2: Gain of function (activating) mutation in ABCC8 or KCNJ11 (KATP channel).

\section{Gain of Function mutation in ABCC8 or KCNJ11 (KATP channel)}

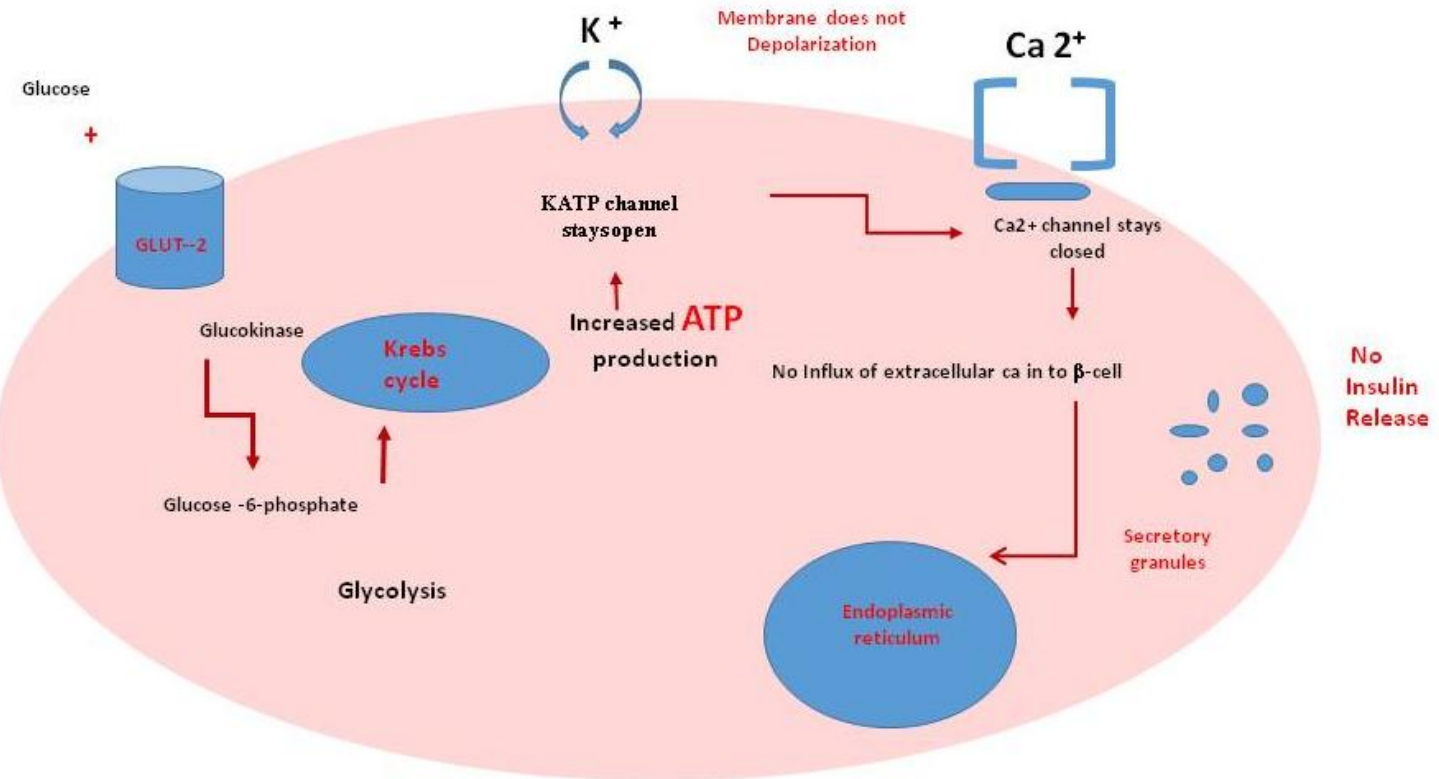

KATP channel remains open with efflux of $\mathrm{K}^{+}$No membrane depolarization. No influx of calcium and no insulin secretion. 
Figure 3: Loss of function mutation in ABCC8 or KCNJ11 9(KATP channel) results inreduction or absence of protein at the membrane.

\section{Loss of Function mutations in ABCC8 or KCNJ11 (KATP channel) It prevents trafficking KATP channel}

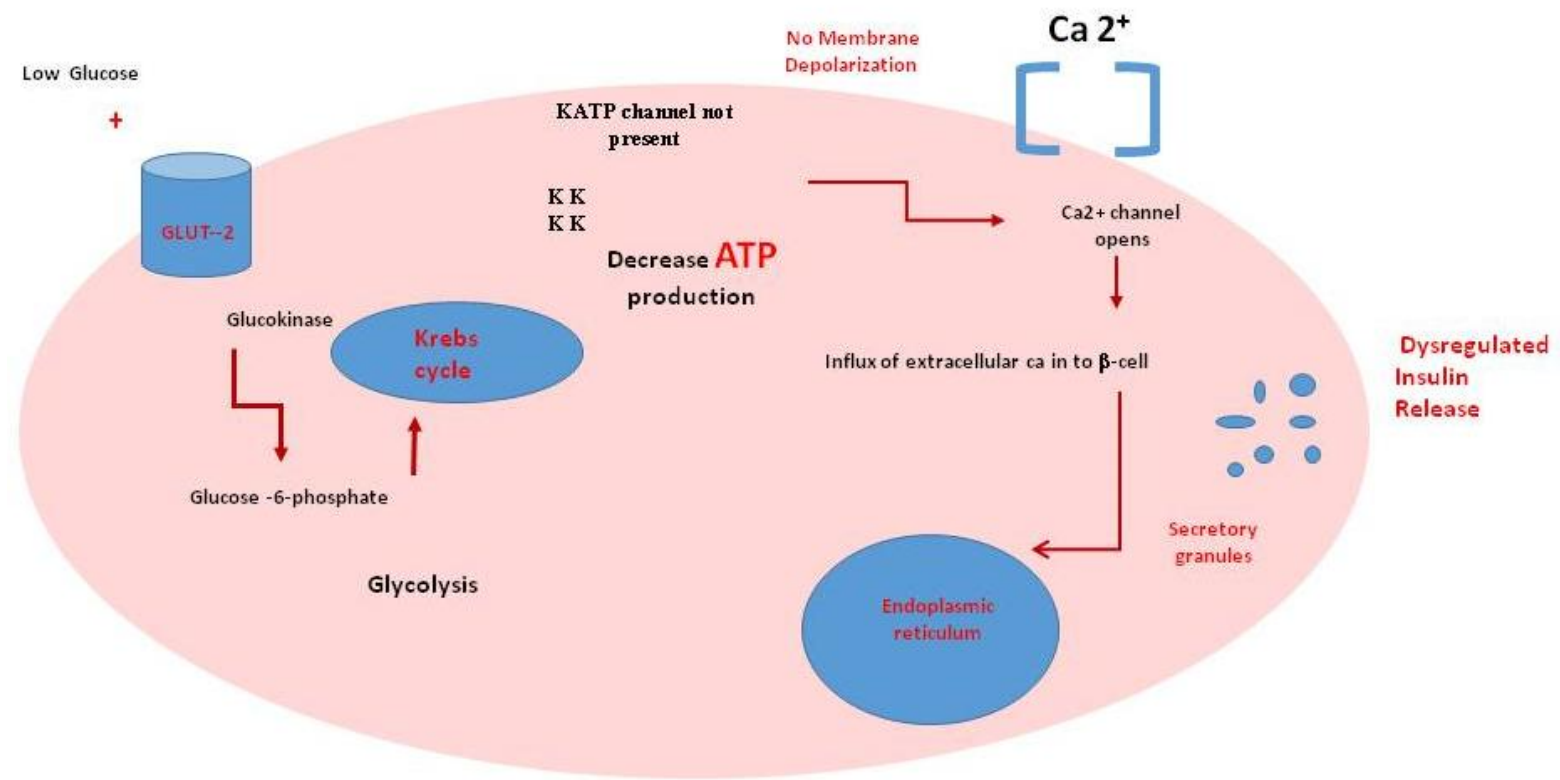

Figure 4: Loss of function mutation in ABCC8 or KCNJ11 (KATP) with reduced responsiveness of KATP to mgADP.

\section{Loss of Function mutations in ABCC8 or KCNJ11 (KATP channel) With reduced responsiveness of KATP to $\mathrm{mgADP}$}

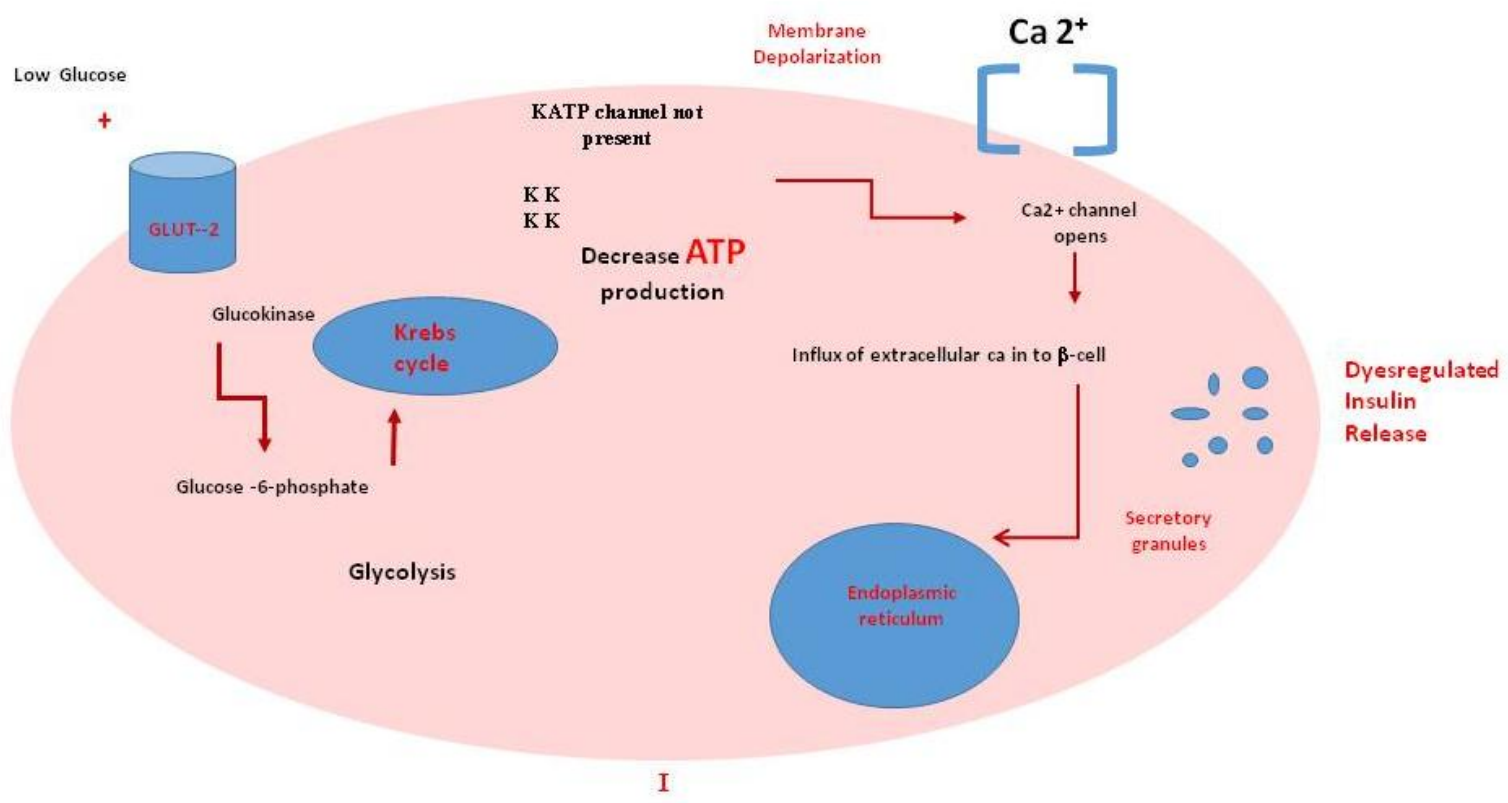


Despite our current understanding, it is also likely that the KATP channelindependent mechanisms of $\beta$-cell insulin release also exist, and the ongoing research will help to understand this further. This may explain the variable penetrance and presentation of diabetes within the same family that ranges from neonatal hypo/hyperglycemia, remission of neonatal hyperglycemia with relapse in early adulthood, the first presentation in adulthood, or only as gestational diabetes or glucose intolerance. The potential for misdiagnosing monogenic diabetes as either late onset Type 1 or early Type 2 still exists, and could result in mismanagement. These patients often do not achieve optimal glycemic control with therapies used for common forms of diabetes and are prone to serious risks of hypoglycemia. Without the right diagnosis, they may also never achieve a good quality of life.

$\mathrm{ABCC} 8 / \mathrm{KCNJ}$ [11] mutations are easy to treat with sulfonylurea, or may not require medical therapy in some cases depending on the type of pathogenic variant. If identified early with a detailed history, particularly family history and cascade genetic testing, these patients can have appropriate follow up with prompt intervention and treatment when clinically indicated.

\section{Case Report}

A 58-year-old lady was referred for management of her diabetes by the Genetics department to the inherited diabetes clinic after a Heterozygous novel missense mutation was detected in the ABCC8 gene during cascade screening. She was diagnosed as Type 2 diabetes incidentally from a routine screening at school when she was 12 years of age. She was managed with diet alone for several years following the diagnosis. She was treated with insulin for optimization of glycemic control during preconception between 2002 and 2003. Insulin was discontinued, and Gliclazide was prescribed after 2003. However, she experienced several episodes of hypoglycemia, in particular, post-exercise, and eventually, Gliclazide was discontinued in 2013. Her past medical history includes appendicectomy, vitamin deficiency, and hyperlipidemia. Her current medication is only Vitamin D and Atorvastatin. She does not smoke, drinks 12 units of alcohol/week, and leads an active lifestyle, which includes regular swimming and cycling sessions. Several members of her family have developed diabetes in their twenties spanning over four generations, as depicted in the pedigree chart Figure: 5 .

Figure 5: Pedigree Tree.
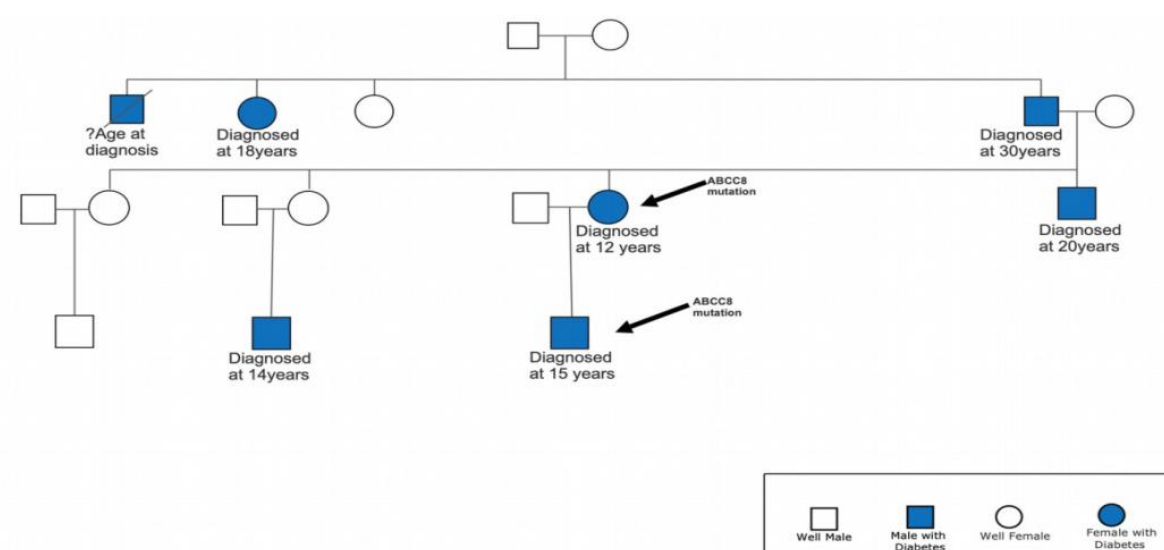
Clinical examination revealed a shortstatured female with a height of $149.5 \mathrm{~cm}$, a body weight of $46 \mathrm{~kg}$ with a BMI of 20.5 . Her $\mathrm{HBAlC}_{1} \mathrm{C}$ has ranged between 42 to $51 \mathrm{mmol} / \mathrm{mol}$. Her Total cholesterol was $5.4 \mathrm{mmol} / \mathrm{L}$, Triglycerides $0.67 \mathrm{mmol} / \mathrm{L}$, HDL cholesterol $3.21 \mathrm{mmol} / \mathrm{L}$ and Cholesterol/HDL ratio- 1.7. Her other investigations, including urine albumin creatinine ratio (ACR), renal ultrasound, and the annual diabetic eye screening, were unremarkable [10]. She has no diabetes-related micro or macrovascular complications. She has a good diabetes control with diet and exercise alone.

\section{Mutation Result}

\begin{tabular}{|c|c|}
\hline Result & Heterozygous Mutation Identified \\
\hline & $\begin{array}{c}\text { Gene: ABCC8 } \\
\text { Location: Exon } 37\end{array}$ \\
$\begin{array}{c}\text { Mutation } \\
\text { details }\end{array}$ & $\begin{array}{c}\text { DNA Description: c.4522G A } \\
\text { Protein description: p.Ala15o8Thr } \\
\text { (p.A15o8T) } \\
\end{array}$ \\
Consequence: Missense \\
\hline
\end{tabular}

Her son was the first in the family to be diagnosed with this pathogenic variant in the ABCC 8 gene at the age of 16 years. He is currently 23 years old. He was born prematurely at 34 weeks with a low APGAR score.

His birth weight was $2.7 \mathrm{~kg}$ (large for age), he had recurrent neonatal hypoglycemia and cerebral palsy. His hypoglycemia at birth was managed with frequent feeds and Diazoxide. Hypoglycemic episodes were transient, and he was in remission until 15 years of age. He then presented with hyperglycemia. Due to the significance of his neonatal history, genetic testing was undertaken, and further cascade screening has been undertaken in his first degree relatives.

\section{Discussion}

\section{Potassium ATP Channel}

The potassium sensitive ATP channel is a hetero-octamertrans membrane channel. It has four SUR1- sulfonylurea receptors that regulate four, pore-forming Kir 6.2 (inward-rectifier potassium ion channels) Figure 6. They were first identified in cardiac muscle in the 1980 os and were subsequently found to be present in skeletal muscle, smooth muscle, pancreatic beta cells, and neurons. Sulfonylurea components of the channel were discovered a decade later, and it was realized that the co-expression of SUR1 receptor proteins is essential in sensitizing the pore-forming potassium channel to nucleotides like ATP/ADP and sulfonylureas [10]. On binding with ATP, the channel closes, causing membrane depolarization and opening of voltagegated calcium channels, this results in extracellular calcium ions influx into the, which eventually causes exocytosis of insulin and normalization of blood glucose levels 7 (Figure.1).

Figure 6: The structure of the KATP channel.

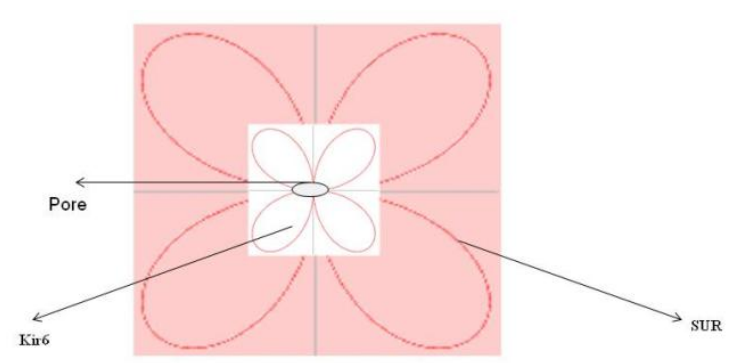

The KATP channel is a hetero- octamer composed of Sulfonylurea recetor (SUR) and 4 Kir6 subunits [3] are centrally located and form the pore and SUR are peripherally 
The gating mechanisms that are involved in the opening and closing of KATP channels can be ligand dependant or independent. The ligand-independent mechanisms are not clearly understood and are thought to be a result of the recruitment of protein kinases $A$ and $C$, changes in membrane potential, or a state of selectivity filter 5 that allows potassium transport despite metabolic activity within the cell.

On the other hand, ligand dependant mechanisms involve PIP2 (phosphatidylinositol 4, 5 bisphosphates) and Nucleotides. The binding of PIP2 and presence of Magnesium ions (causing conversion of MgATP to MgADP) activates and opens the gate, whereas the binding of ATP (in the absence of Mg2+ ions) causes channel closure Figure 7 and Figure 8 . There are four Kir subunits, but the binding of even one subunit with ATP is enough to cause the closure [25]. This mechanism is not clearly understood and it could be that the ATP binding to all four subunits acts in a concerted way or independent of each other $[8,9]$.

There are three forms of the sulfonylurea receptor, SUR1 encoded by the ABCC 8 gene, and SUR2A and SUR2B, which are splice variants arising from a single $\mathrm{ABCC}_{9}$ gene.

Figure 7: With the closed ATP channel the interaction between 1 Kir6 and 1 SUR subunit.

\section{Interaction between $1 \mathrm{Kir} 6$ and 1 SUR subunit with ATP channel closed.}
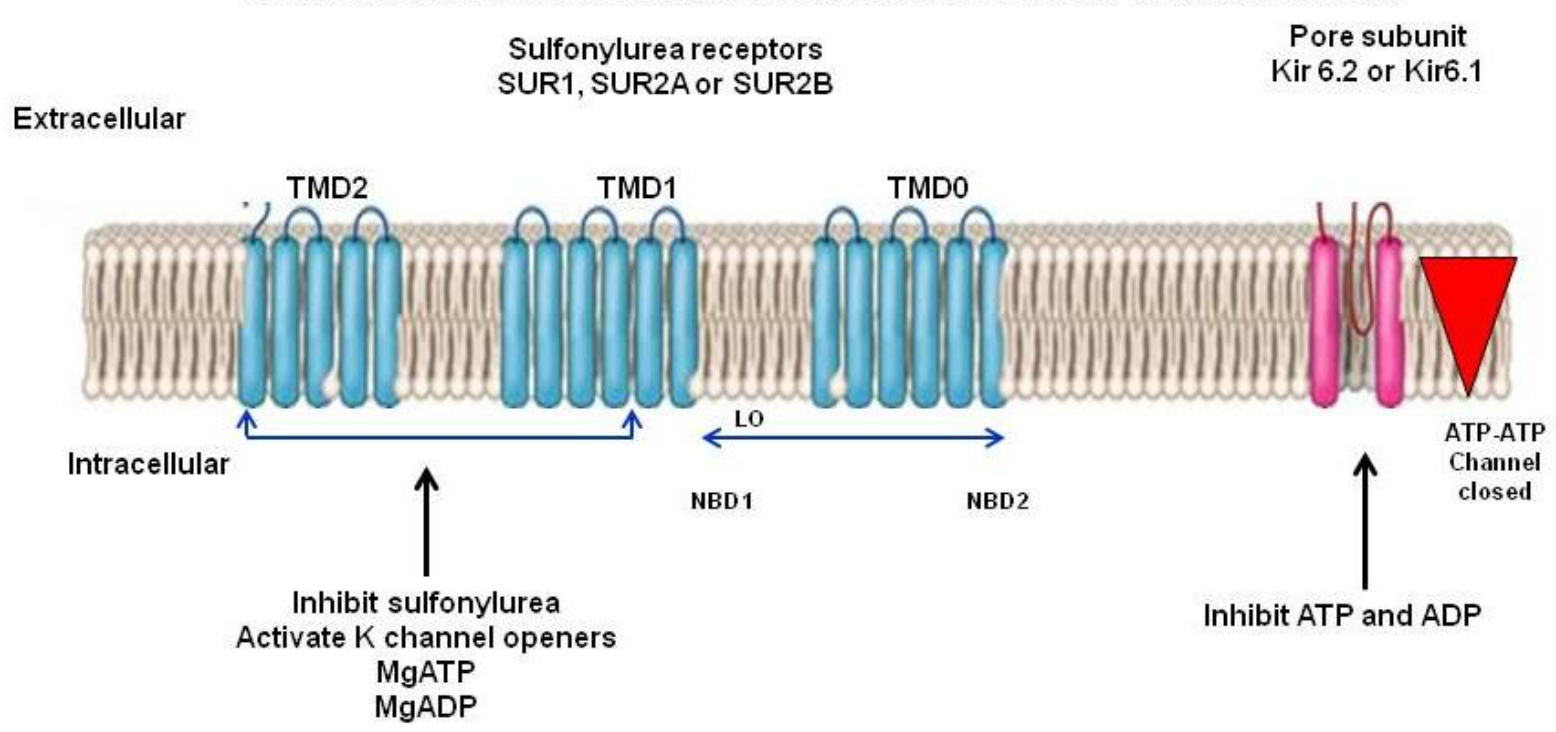

SUR has 3 transmembrane Domains (TMDs), TMDo, TMD1 and TMD2 and 2 Nucleotide binding Domains (NBDs) NBD1 and NBD2. The interaction between SUR and Kir takes place via TMDo and a linker region called Lo localised between TMDs. ATP is bound to NBD1 and NBD2 when KATP channel is closed so no efflux of $\mathrm{K}$. ATP inhibits Kir subunit and stops opening of KATP channel
Upon hydrolysis of ATP and formation of $\mathrm{ADP}, \mathrm{NBD}_{2}$ binds to mgADP The binding is signalled to Kir through the TMDo and Lo region of SUR This stops the inhibitory effect of ATP on the Kir and results in opening of $\mathrm{K}$ channel with influx of calcium and release of insulin.

\section{ABCC8 and KCNJ11 gene}


$\mathrm{ABCC} 8$ gene belongs to the $\mathrm{ABC}$ group of genes which encodes ATP binding cassette transporters. KATP is one such transporter. It encodes the SUR1 protein part of the Potassium channel [10]. A BCC8 gene is located on chromosome 11p15.1 and has 39 exons translating into 1581 or 1582 amino acid protein. KCNJ11 gene also has the same chromosomal location as ABCC8 but only has one exon and translates into a 390 amino acid protein (Kir 6.2 Protein).

Figure 8: With ATP channel open the interaction between 1 Kir6 and 1 SUR subunit.

\section{Interaction between 1 Kir6 and 1 SUR subunit with ATP channel open}

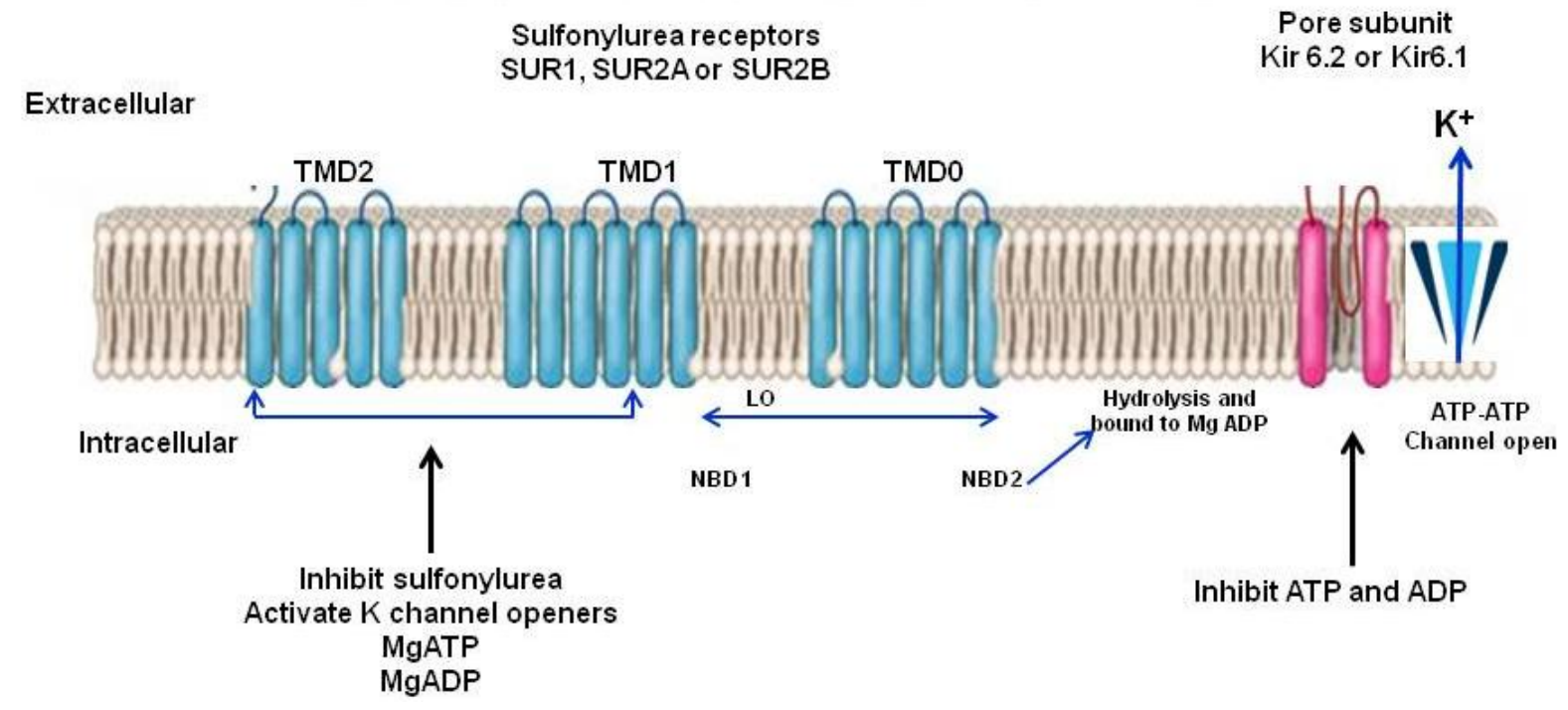

Both Autosomal dominant and recessive mutations in the $\mathrm{ABCC} 8 / \mathrm{KCNJ} 11$ gene could result in either loss or gain of function. However, the majority of the loss of function mutation is due to recessive mutations in the $\mathrm{ABCC} 8 / \mathrm{KCNJ} 11$ gene. This causes either a faulty KATP channel that does not migrate to the cell membrane or KATP channel that incorporates into the cell membrane but is less responsive to MgADP stabilization of the channel [10] This results in no potassium efflux across cell membrane, and the continued depolarization of the membrane, that leads to dysregulated and persistent Insulin release and hypoglycemia in the neonatal period. A few dominant missense mutations have been described, which results in KATP channels that traffic to the cell surface normally but are less sensitive to $\mathrm{Mg}+\mathrm{ADP}$ Figure 3 and Figure 4 . These are responsive to Diazoxide therapy. Some dominant mutations have also been reported with faulty manufacture of the KATP channel that does not reach the cell membrane and hence remains resistant to medical therapy [9].

A gain of function mutation results in the KATP channel that is less responsive to ATP with the resultant KATP channel that is continuously open Figure 2. These patients may present with transient or permanent neonatal diabetes or onset of diabetes later in life [2]. This variable penetrance is still not clearly understood, and could also be related to KATP independent mechanisms of Insulin release from the $\beta$-cell, which is not yet identified. Both dominant and recessive ABCC 8 
mutations and most dominant mutations of KCNJ11 gene have been associated with the gain of function [10].

Both our patient and her son have a novel pathogenic variant that has not been described before.

As in our index case, the biphasic presentation of neonatal hypo/hyperglycemia, remission in early childhood followed by diabetes later in life has been described in $\mathrm{ABCC} 8 / \mathrm{KCNJ} 11$ mutations. This is associated with dominant mutations, and the underlying mechanisms for this are not understood. Laboratory study of mouse models with dominant homozygous loss of function mutation showed a similar biphasic picture of hyperinsulinemichypoglycemia followed by diabetes later.

This was related to a decrease in Insulin content despite normal $\beta$-cell volume. However, a heterozygous mutation in mice did not result in a disease [11].

\section{Clinical Manifestations}

ABCC8/KCNJ11 mutations could present with any or combination of the following:

\section{Congenital Hyperinsulinemic Hypoglycemia (CHH) [19]}

This is a standard name of the condition not able to change. This is a classic presentation of loss of function mutation of KATP channel where there is continued depolarization of cell membrane leading to persistent Insulin release and severe hypoglycemia. This could be a result of the KATP channel not reaching the cell membrane, or the channel is unresponsive to MgADP. Babies are typically born with large birth weight and may develop severe hypoglycemia and a neurological sequel if undiagnosed. $\mathrm{CHH}$ is further subdivided into focal, diffuse, and atypical variety depending on the histological features. Commonest is diffuse variety, where all the $\beta$ cells of the pancreas are affected. This is often a result of compound heterozygous or homozygous recessive mutation [10]. Focal variety presents with localised islet cell hyperplasia and is due to paternally inherited heterozygous mutation [10] The focal variety is due to lack of balanced expression of IGF-2 (Insulin-like growth factor-2) and $\mathrm{H}_{19}, \mathrm{CDKN}_{1} \mathrm{C}$ (tumor suppressor genes). An atypical type constitutes poorly defined histology that does not fit into a focal or diffuse variety. This type is not clearly understood, and no clear, mutations have been identified so far.

\section{Neonatal Diabetes}

Patients can present with transient or permanent neonatal diabetes [1]. Activating heterozygous mutations of both ABCC 8 and KCNJ11 genes have been detected in these patients. A study conducted on 97 patients who were diagnosed with diabetes before six months of age documented that the transient neonatal diabetes resulting from KATP channel mutations always occurs before six months of age, remits in early childhood and $50 \%$ of cases relapse in adolescence or early adult life.

About $71 \%$ of these patients had chromosome 6 q24 abnormality- the commonest genetic cause of neonatal diabetes and $26 \%$ had KATP channel abnormalities [7]. This study also showed that those cases who did not have remission after six months would go on to have permanent neonatal diabetes. This is as a result of gain of function mutation [7]. T2 Diabetes that presents in the first six months of life is most likely due to a genetic mutation (because Type 1 diabetes 
does not present this early. Patients born KATP channel mutations generally have low birth weight) and are easily treated with oral sulfonylurea.

3. Diabetes in adolescence or early adult life: (Unable to change the name, this is a standard name)

It is rare to find $A B C C 8 / K C N J 11$ mutations in patients presenting with diabetes for the first time in early adult life without a history of neonatal hypo or hyperglycemia [5]. The majority of these patients are dominant mutation carriers presenting with any of the presentations, glucose intolerance, or gestational diabetes or diabetes in early adult life or no diabetes at all and might simply pass the gene to offspring who may present with variable penetrance of diabetes. This implies that there are undiscovered pathways involved in $\beta$-cell insulin release besides the KATP channel.

\section{Neurological Manifestations}

It is well known that KATP channels are expressed in neural tissue. Nearly 30\% of the patients [2] presenting with permanent neonatal diabetes was noted to have concomitant neurological features. The neurological symptoms range from autistic spectrum disorders, neuropsychological abnormalities, sleep disorders, DEND syndrome (Developmental delay, epilepsy, neonatal diabetes), or as intermediate DEND (IDEND) syndrome where epilepsy is either not present or appears after 12 months of life [11, 12, 13].

\section{When to suspect Monogenic Diabetes?}

Features suggestive of clinical diagnosis of MODY: Onset of diabetes in adolescence or young adulthood typically before the age of 35 years.
Features not typical of type 1 diabetes. These include:

No detection of pancreatic islet autoantibodies

Evidence of endogenous insulin production beyond honeymoom period ( 3-5 years after the onset of diabetes)

In presence of hyperglycemia measurable C-peptide (C-peptide $\geq 0.60 \mathrm{ng} / \mathrm{mL}$ or $0.2 \mathrm{nmol} / \mathrm{L}$ )

Requirement of insulin $0.5 \mathrm{U} / \mathrm{kg} / \mathrm{d}$ (low)

Omission of insulin does not cause ketoacidosis

Features not typical of type 2 diabetes mellitus. These include:

Age of onset of diabetes before 45 years

No features of insulin resistance

Not obese

No acanthosisnigricans

No evidence of fatty liver

Normal or elevated high-density lipoprotein cholesterol (HDL-C) and normal triglyceride levels

Non progressive mild, stable fasting hyperglycemia that responds to pharmacologic therapy

Highly sensitive to sulfonylureas

Extrapancreatic manifestations (e.g [1] renal, hepatic, gastrointestinal)

Personal history or family history of neonatal diabetes or neonatal hypoglycaemia. 
There should be a high index of suspicion about monogenic diabetes in a child with an affected parent with diabetes, and particularly if there is $\geq 3$ consecutively affected generations [5].

$60-90 \%$ of MODY will have an affected parent compared to $10 \%$ of those with Typer Diabetes [5]. However, a history of parent with diabetes alone cannot differentiate between Type $\mathbf{2}$ diabetes (T2D) and MODY. In young onset type 2 cases 50\% will have a parent with diabetes. Suspicion should increase if the patient is not obese or has no metabolic features.

Biomarkers that can help in the selection of individuals for monogenic diabetes genetic testing include C- peptides, pancreatic auto-antibodies, lipid profiles, and highsensitivity C-reactive protein (CRP) which has a discriminatory variable value with several limitations.

A combination of clinical and biomarker approaches is likely to yield the highest sensitivity [5]. Absence of pancreatic autoantibodies in a non-obese patient with no metabolic features and a detectable Cpeptide 3-5 years after diagnosis increases the clinical suspicion.

In the US SEARCH study, youth diagnosed under the age of 30 with diabetes was selected for the genetic testing if pancreatic autoantibody test was negative and fasting C-peptide was $0.8 \mathrm{ng} / \mathrm{ml}$. This method yielded a detection rate of $8 \%$ in the tests $[5,15]$.

Availability of an online clinical prediction tool for MODY has facilitated the calculation of an individual's probability of having MODY. This also provides a rational approach to genetic test. The disadvantage is that this tool kit only applies to individuals younger than age 35 years and has been mostly studied in white European populations. The prevalence of MODY in racial and ethnic minorities may be underrepresented as many individuals with MODY remain undiagnosed [16].

\section{Management}

\section{Congenital Hyperinsulinemic Hypoglycemia:}

Diazoxide therapy is useful in patients with congenital hypoglycaemia [21]. Diazoxide acts through SURıreceptor, causing activation of the KATP channel that results in opening of the KATP channel, hyperpolarisation and a decreased Calcium ion influx, thus counteracting Insulin release. In patients who show poor response to Diazoxide, Somatostatin analogs have been tried as the second line with beneficial effects. Poor response to medical therapy depends on the type of defect in the KATP channel (when the KATP channel does not traffic to the cell membrane from endoplasmic reticulum) and severity of the mutation.

18F-DOPA-PET (fluorine-18 dihydroxyphenylalanine positron emission tomography) scan can distinguish between focal and diffuse types of $\mathrm{CHH}$. Surgical resection of focal abnormality can result in the resolution of symptoms. However, these patients may still develop diabetes later in life due to reduced expression of proinsulin secretion by the remaining pancreatic cells. The diffuse type does not respond well to medical therapy and may need total pancreatectomy that results in diabetes and exocrine pancreatic insufficiency.

\section{Diabetes}

Sulfonylureas are the drug of choice in both transient/permanent neonatal diabetes and also for diabetes that develops later in life. This drug is well tolerated, and not many serious adverse effects in terms 
of hypoglycemia have been reported so far [8]. Sulfonylurea binds to the SUR1 receptor of the KATP channel and closes the channel independently of ATP, resulting in insulin release. The cause for the unresponsiveness to sulfonylurea therapy is due to the duration of diabetes, severity of the mutations, and duration of Insulin therapy prior to the transfer of therapy from insulin to sulfonylurea following a revision of the diagnosis. In patients who have concomitant neurological features, larger doses of sulfonylurea are recommended, and treatment initiation should be as early as possible because there is some evidence that this may improve the neurological features.

Permanent neonatal diabetes (PND) caused by mutations in $\mathrm{KCNJ} 11$ or $\mathrm{ABCC} 8$ can be managed with high- dose sulfonylurea therapy in $90 \%$ of cases. For neonatal diabetes, a recent case series of a 10-year follow- up of individuals with KCNJ11 or $\mathrm{ABCC} 8$ variants treated with $\mathrm{SU}$ agents reported that $93 \%$ of cases remained on SU therapy alone, achieving glycemic targets and with a good safety profile.

\section{Pregnancy}

Management of patients with monogenic diabetes and Pregnancy is challenging. Factors that need to be considered in these patients are: Maternal Diabetes control, mutation status of the fetus, Effectosulfonylurea and its transplacental transfer of sulfonylurea [10]. Treatment in a non-pregnant state is mostly with sulfonylureas, but, during pregnancy, the benefit of sulfonylurea treatment will depend on the fetal genotype.

Fetal Genotype determines the fetal birth weight in the pregnancy affected by the KATP channel mutation. Those who inherit the mutation from either their father or mother can have low birth weight due to reduced fetal insulin secretion; Sulfonylurea treatment will restore the fetal insulin secretion in such cases and can result in normal birth weight. On the other hand sufonylurea treatment can cause excess fetal insulin secretion, macrosomia and neonatal hypoglycaemia if the fetus has not inherited the mutation.

The risk from deteriorating glycemic control in the mother on a high dose of sulfonylurea and its effect on the fetal development has to be accounted for. This can again result in macrosomia and neonatal hypoglycemia in an unaffected offspring, who is being exposed to high dose sulfonylurea treatment. Chorionic villus sampling or amniocentesis can be used for fetal genotyping. These ivasive procedures can cause miscarriage [10]. Noninvasive cell-free DNA is an option for prenatal genetic testing. This could help management strategies for many forms of monogenic diabetes [10].

As per current recommendations fetal genotype can be inferred from serial ultrasound growth monitoring starting at 28 weeks [10]. Reduced growth indicates the fetus has inherited the KATP channelmutation, while normal growth suggests no mutation.

[10] General treatment guidelines advise to switch to insulin prior to conception or to continue sulfonylurea treatment through the first trimester at the lowest dose to maintain good glycemic control with HBAic 6.5.

If ultrasound scans show reduced fetal growth, indicating that the fetus has inherited the KATP channel mutation, sulfonylurea (glyburide) is the treatment of choice and should be continued or 
reintroduced if the patient was switched to insulin previously.

If ultrasound scans show normal fetal growth then transfer from sulfonylurea to insulin in order to avoid excessive fetal growth, macrosomia, and neonatal hypoglycemia.

Fetal genetic testing should be done after delivery if it was not done during pregnancy [10]. If the offspring has inherited the KATP channel mutation, neonatal diabetes will present before 6 months of age. A regular follow up with a pediatrician is recommended [10]. Maternal sulfonylurea treatment can be restarted after delivery and is considered to be safe during breast feeding, though at high maternal doses, may be excreted in the breast milk $[17,18,19]$.

\section{Conclusion}

The importance of history taking and arriving at a diagnosis of monogenic diabetes cannot be stressed enough as it carries huge therapeutic implications. The case described is a classic example of delayed diagnosis of monogenic diabetes and inappropriate use of Insulin therapy resulting in dangerous hypoglycemia. The chances of the patient's son being treated as Type 1 diabetes with Insulin therapy were high without clinical suspicion of monogenic diabetes.

This would have resulted in the challenging management of a 15-year-old patient with cerebral palsy. The implications of a diagnosis of diabetes in childhood and adolescence are profound. Cascade testing the family members with diabetes and a successful transfer from Insulin therapy to sulfonylurea will improve glycemic control effectively, but also results in a significant decrease in disease burden.

Several members of the patient's family have developed diabetes in their twenties, with the youngest being the patient's son. Although the patient and the son share the same novel pathogenic variant, the phenotype and presentation of the patient are very different from the son who has severe neurological features and a biphasic presentation of diabetes.

This variable penetrance of diabetes can imply that the KATP channel may not be the only responsible pathway involved in Insulin release. Further research studies are required to explore the molecular mechanisms responsible for $\beta$-cell Insulin release other than KATP channel pathways. Even within the KATP channel, it is not clear how the four Kir 6.2 subunits are orchestrated, as the binding of even one subunit with ATP is enough to result in the closure of the channel.

The current challenges in monogenic diabetes are lack of awareness and understanding of monogenic diabetes among medical practitioners, access to high quality and cost-effective genetic testing, and lack of ethnic studies, as the majority of data available are based on the population of the European descent [8].

Research is needed across all the ethnic groups with contributions from national and international data.

\section{References}

1. Misra S, Owen KR. Genetics of Monogenic Diabetes: Present Clinical Challenges. Curr Diab Rep. 2018; 1:141.

2. Frances M Ashcroft, and Patrik Rorsman. Diabetes mellitus and the $\beta$-cell: the Last Ten Years. Cell.2012; 148: 1160-1171. 
3. Ellard S, Bellanne-Chantelot C, Hattersley AT and European Molecular Genetics Quality Network (EMQN) MODY group. Best practice guidelines for the molecular genetics diagnosis of maturityonset diabetes of the young. Diabetologia. 2008; 51: 546-553

4. De Franco E, Saint-Martin C, Brusgaard $\mathrm{K}$, et al. Update of variants identified in the pancreatic $\beta$-cell KATP channel genes KCNJ11 and ABCC8 in individuals with congenital hyperinsulinism and diabetes. Hum Mutat. 2020;41:884-905.

5. Naylor R, Johnson AM, and Daniela del Gaudio D. Maturity-Onset Diabetes of the Young Overview. GeneReviews ${ }^{\circledR}$ [Internet]. Seattle (WA): University of Washington, Seattle; 1993-2020. 2018

6. Donathl X, Saint-Martin C, DuboisLaforgue $\mathrm{D}$ et;al. Next-generation sequencing identifies monogenic diabetes in $16 \%$ of patients with late adolescence/adult-onset diabetes selected on a clinical basis: a crosssectional analysis. BMC Medicine.2019; 17:132

7. Burke MA, Mutharasan RK, Ardehali H. The Sulfonylurea Receptor, an Atypical ATP-Binding Cassette Protein, and Its Regulation of the KATP Channel. Circ Res. 2008; 102:164-176

8. Rahman S, Nessa A, \& Hussain K. Molecular mechanisms of congenital hyperinsulinism. Journal of Molecular Endocrinology. 2015; 54: R119-R129.

9. Nessa, Azizun et al. "Hyperinsulinemic Hypoglycemia - The Molecular Mechanisms." Frontiers in endocrinology. 2016; 7: 29.

10. Quan Y, Barszczyk A, Feng Z and Sun $H$. Current understanding of KATP channels in neonatal diseases: focus on insulin secretion disorders. Acta Pharmacol Sin. 2011; 32:765-780.

11. Shimomura, K, Tusa, M. , Iberl, M.et.al. A mouse model of human hyperinsulinism produced by the E1506K mutation in the sulphonylurea receptor SUR1. Diabetes. 2013; 62: 3797-3806.

12. Flanagan SE, Patch AM, Mackay D et.al,. Mutations in ATP-Sensitive $\mathrm{K}+$ Channel Genes Cause Transient Neonatal Diabetes and Permanent Diabetes in Childhood or Adulthood. Diabetes 2007; 56: 1930-1937.

13. Kapoor RR, FlanaganSE, James CT et.al,. Hyperinsulinaemic hypoglycaemia and diabetes mellitus due to dominant ABCC8/KCNJ11 mutations. Diabetologia.2011; 54:2575-2583

14. Ludvigsson J, Carlsson A, Forsander G et; al. C-peptide in the classification of diabetes in children and adolescents. Pediatric Diabetes 2012; 13:45-50

15. Hamman RF, A. Bell RA, Dabelea D et.al, The SEARCH for Diabetes in Youth Study: Rationale, Findings, and Future Directions Diabetes Care 2014;37:33363344 .

16. Shields BM, McDonald TJ, Ellard S, Campbell MJ, Hyde C, and Hattersley AT. The development and validation of a clinical prediction model to determine the probability of MODY in patients with young-onset diabetes. Diabetologia. 2012; 55: 1265-1272.

17. Sanyoura M1, Philipson LH, and Naylor R1. Monogenic Diabetes in Children and Adolescents: Recognition and Treatment Options. Curr Diab Rep. 2019; 1-20 
18. Dickens LT, Naylor RN. Clinical Management of Women with Monogenic Diabetes During Pregnancy Curr Diab Rep. 2019; 18:1-15

19. Bowman P, Sulen A, Barbetti F, Beltrand J, Svalastoga P, Codner E, et al.
Effectiveness and safety of long-term treatment with sulfonylureas in patients with neonatal diabetes due to KCNJ11 mutations: an international cohort study. Lancet Diabetes Endocrinol. England. 2018; 6:637-46. 